\title{
Effect of Combined Training on Body Image in Women with Breast Cancer: Controlled Clinical Trial
}

Andréa Dias Reis ( $\square$ adr.dea@hotmail.com )

Universidade Estadual Paulista Julio de Mesquita Filho https://orcid.org/0000-0002-1881-4382

Paula Tâmara Vieira Teixeira Pereira

Universidade Federal do Maranhão

Jurema Gonçalves Lopes Castro Filha

Universidade Federal do Maranhão

Evelyn Feitosa Rodrigues

Universidade federal do Maranhão

Isadora Pinheiro Laranjeira

Universidade Federal do Maranhão

Bianca Trovello Ramallo

Universidade Sao Judas Tadeu

Marcela Rodrigues de Castro

Universidade Federal da Bahia

Fabrício Eduardo Rossi

Universidade Federal do Piaui

Ismael Forte Freitas Júnior

Universidade Estadual Paulista Julio de Mesquita Filho

João Batista Santos Garcia

Universidade Federal do Maranhão

Research article

Keywords: Breast Neoplasms, Body Image, Exercise, Women, Body Composition, Physiology

Posted Date: July 28th, 2020

DOI: https://doi.org/10.21203/rs.3.rs-42441/v1

License: (c) (i) This work is licensed under a Creative Commons Attribution 4.0 International License. Read Full License 


\section{Abstract}

Background: Improvement in body image in women with breast cancer may be linked to altered functional capacity, regardless of body composition. The aim of this study was to evaluate the effect of combined training (CT) on body image in patients with breast cancer.

Methods: A Clinical Trial study, this was a controlled study including 26 patients with breast cancer (30 to 59 years). The patients were allotted to either the Training Group $(T G)(n=13)$, which underwent 12 weeks of CT (aerobic, resistance, and flexibility), or the Control Group (CG) $(n=13)$, which received only the standard hospital treatment. Participants were evaluated at baseline and after 12 weeks.

Results: The patients in the TG showed a significant reduction in the limitation dimension $(p=0.036)$, and an increase in V02max $(p=<0.001)$ and strength in the right $(p=0.005)$ and left arms $(p=0.033)$. However, the participants also presented an increase in waist circumference $(p=0.034)$.

Conclusion: Women with breast cancer presented improved body image and functional ability through CT.

Trial registration: NCT03061773. Date: February 23, 2017, retrospective.

\section{Background}

According to the World Health Organization [1], at least $16 \%$ of the world's population died of cancer in 2015 , with breast cancer being the most frequent in women, especially in underdeveloped countries[2]. In Brazil, breast cancer was estimated 59.700 new cases of the female population, representing 720 new cases in the state of Maranhão, the site of this research [3].

The disease and its treatment can generate important changes in body appearance and functionality. Changes in appearance include alopecia, surgical scars, breast removal, rashes etc. [4, 5]. Mastectomy can lead to emotional, social, postural, and sexual alterations. It can also cause lesions in muscles, lymphedema, and a decrease or loss in range of motion. Furthermore, patients on antineoplastic therapy may present reduced strength and cardiopulmonary capacity[6].

Body composition is considered a worrying factor, since treatment may imply a weight gain of 71.43\%[7]. Excess weight and obesity are a poor prognosis, due to increases in tumors, positivity of estrogen and progesterone receptors, risk of distant metastasis, and mortality[8].

According to Muzzatti and Annunziata (2017), there has been an expansion in the clinical and investigative interest in oncology since 2012 [9], when Supportive Care in Cancer highlighted the importance of assessing body image in cancer patients. Body image is a multidimensional construct, and it is necessary to consider the subjective experiences of the disease[5], as well as the symbolic value attributed to specific body segments, such as the breast, for woman[9]. 
Thus, in the elaboration of body image, the social and cultural influence derived from interpersonal experiences should be considered, allied to several elements of the appearance and body functionality [10]. Therefore, women with breast cancer are vulnerable to adverse impacts on their body image[11, 12, 4], which in turn can generate various consequences such as anxiety, depression, mutilation, and low selfesteem[13], as well as a loss of connection with identity, leading ultimately to social isolation. In addition, adverse changes in body image have the potential to cause difficulties in breast cancer recovery [13].

Evidence supports the need to develop practical intervention strategies directed at the body image of the public in question[12]. Physical training methods, both strength and aerobic, have been used as safe and well tolerated interventions in cancer patients[14-17]. Studies have shown a decrease in body fat, and improvements in cardiopulmonary function, strength $[15,16]$, and body image $[18,19]$ after interventions with physical activity. However, although interventions with combined training (CT), aerobic and resistance training, are widely used [20-22], the available information is still insipid, especially in the Brazilian population [16]. Thus, the present study aims to evaluate the influence of CT on the body image of patients with breast cancer.

The hypotheses were: 1) There would be a significant difference in body composition, function, and image between the training and control groups; 2) Changes in body composition and functionality, promoted by CT, would positively impact the body image of women with breast cancer.

\section{Methods}

\section{Participants}

Thirty-one women (30 to 59 years old) selected from the Aldenora Bello Cancer Hospital in São Luís-MA participated in the study, through standardized invitations given at routine meetings at the institution. The inclusion criteria were: 1) not having performed physical training for at least 6 months, 2) being on treatment (chemotherapy, radiotherapy, and hormone therapy) or monitoring of breast neoplasms, 3 ) no diagnosis of mental disorders or psychological disorders, 4) able to communicate verbally, 5) no motor restrictions, 6) not pregnant or lactating, 7) performing all evaluations, 8) having previous medical release. To remain in the physical training group, it was necessary to not be absent from more than three consecutive sessions.

\section{Allocation}

Groups were divided 1:1, they were randomly assigned to groups into the Training Group (TG), with 15 patients who performed CT for 12 weeks together with conventional hospital treatment, and the Control Group (CG) with 16 patients who only underwent conventional hospital treatment for 12 weeks.. The Term of Free and Informed Consent was applied to all patients in the study.

\section{Ethical permission and Clinical trial}


Participants were informed about the objectives of the study and written informed consent was obtained.

The study had approval from the Research Ethics Committee of the Federal University of Maranhão, protocol number 20665713.2.0000.5087 and Trial Registration: NCT03061773.

\section{Primary outcome}

\section{Body Image}

Body image was assessed using the Body Image After Breast Cancer Questionnaire (BIBCQ)[23], a specific instrument for patients with breast cancer, which includes items directed to mastectomized women without breast reconstruction, with conservative surgery, or with mastectomy and breast reconstruction. This tool was validated for the Brazilian female audience by Gonçalves et al. [5]. It consists of 44 questions organized in 6 dimensions: 1) Vulnerability (V), 2) Transparency (T), 3) Body Stigma (BS), 4) Concerns about the Arm (CA), 5) Body Concerns (BC), and 6) Limitations (L). The answers are given on a Likert scale of agreement, assigning values from 1 to 5 . The scores vary according to the scale and surgery; the higher the score, the more compromised the body image[24, 5]. For the questions that presented negative scores, a value of 6 is inserted for the calculations of the dimensions [25].

\section{Secondary outcomes}

\section{Body Composition}

Body composition was verified through the following indicators: 1) Body Mass Index (BMI) = body mass $(\mathrm{Kg}) /$ height $(\mathrm{m})^{2}$ [26]; 2) Weight (kg) [29]; 3) Waist Hip Ratio (WHR) = (Waist Circumference (WC) (cm)/hip circumference(cm))[27]; 4) Waist Height Ratio (RCEST): (Waist circumference $(\mathrm{cm}) / \mathrm{height}(\mathrm{cm})$ ) [27]; 5) Conicity Index (C Index) $(\mathrm{WC}(\mathrm{m}) / 0.109$ square root: weight( $\mathrm{kg}) /$ height $(\mathrm{m}))$ [27]; 6) Reciprocal Ponderal Index $(\mathrm{RPI})=($ height $(\mathrm{cm}) /$ cubic root of body weight $(\mathrm{kg})$ ) [28]; 7) Percentage of Fat (\%F) for women $=((5.03 / \mathrm{BD})-4.59] * 100$ and the Body Density $(\mathrm{BD})=1.0994921-0.0009929(\mathrm{X} 1)+$ $0.0000023(\mathrm{X} 1)^{2}-0.0001392(\mathrm{X} 2)$ ), where BD: Body Density; $\mathrm{X} 1=\Sigma$ three folds (triceps, supra iliac, and thigh) and X2 = age (years)[29]; 8) Circumference of the abdomen, waist, hip, and right and left thighs were measured using a tape measure (Sanny ${ }^{\circledR}$ Brazil) with a precision of $0.1 \mathrm{~cm}$ [29]; 9) Fat-free mass $(\mathrm{Kg})=($ actual weight - fat weight) [29].

\section{Functional Capacity}

To verify the functional capacity, strength and maximum oxygen volume (VO2max) were considered.

The static force was evaluated using a manual dynamometer (Jamar Sammons Preston) scale from 0 to 100 kilograms. Guidance was provided to press the equipment with maximum force, without flexing the elbow or changing the posture. Three attempts were allowed on both sides (alternately)[29].

The V02max was measured using the submaximal cycle ergometer test, estimated according to the American College of Sports Medicine (ACSM)[26] based on the final power in a protocol of 15 Watts per 
minute, using the formula for women: V02max $(\mathrm{ml}-1 \mathrm{~kg}-1){ }^{\star}=(9.39$ (power in Watts) +7.7 (body weight in $\mathrm{kg})-5.88$ (age in years) $+136.7 * \mathrm{VO} 2 \mathrm{max}(\mathrm{ml}-1 \mathrm{Kg}-1 \mathrm{~min}-1))=$ Divide per $\mathrm{Kg}$. Standard Error Estimated $(\mathrm{SEE})=147 \mathrm{ml} / \mathrm{min}$.

\section{Co-variables}

\section{Anthropometric, demographic and clinical aspects}

Anthropometric measurements were recorded for body mass $(\mathrm{kg})$, height $(\mathrm{cm})$, and age (years) [29]. The level of education, family income (minimum wage value $=\mathrm{R} \$ 788.00)$, employment status, marital status, period without physical training practice (eligibility criteria), treatment and follow-up phase, type of neoplasia, and patient surgery were verified by means of anamnesis.

\section{Intervention}

The CT program consisted of aerobic, resistance, and flexibility exercises lasting 12 weeks, with 3 sessions per week of aerobic and resistance training in the same session (supervised by trainers specialized in physical exercise) and an additional 2 sessions per week of flexibility training.

The flexibility sessions lasted for 20 minutes and were intercalated between sessions of aerobic and resistance training in the daytime period. Familiarization with flexibility occurred over two weeks, with three sessions per week, where each patient was followed to ensure the correct execution of all stretching exercises.

Each aerobic and resistance training session lasted 60 minutes, following the order: 30 minutes on cycle ergometer, hip flexion and extension, shoulder development, Swiss ball squatting, French triceps, and curved paddling.

\section{Familiarization}

Familiarization to the aerobic and resistance training was performed over one week in three sessions, with a cycloergometer load of 15 watts, and the individual's body weight and an elastic band in the execution of 8 to 12 repetitions in the resistance exercises with one minute of recovery between one exercise and the next.

\section{Aerobic training}

The aerobic training was controlled by the training heart rate $(T H R)$ using the formula: $T H R=(Y \times(A-B)+$ $B$ [30], if $Y: \%$ of the desired effort, $A$ : Expected maximum heart rate (measured by cardiorespiratory test) and $\mathrm{B}$ : Resting heart rate (measured at rest).

In the cardiorespiratory test, the ramp protocol adapted by Neil et al. [31] was used on a cycle ergometer (ERGO FIT brand, model ERGO 167-FITC CYCLE), with an initial load of 15 watts during 5 minutes of warm-up, followed by incremental stages of 60 seconds with the addition of 15 watts each stage [31]. After the maximum stage reached, an active recovery of 3 minutes with the initial load was performed, the 
stages had from 70 to 90 rotations per minute. Blood pressure was measured with conventional mercury column apparatus $(\mathrm{BD} \AA)$, heart rate (Polar FT2) and subjective perception of exertion using the Borg scale (PSE) (Inforfisic Mark) in the final 15 seconds of the stages. Before and after the cardiorespiratory test, the patients remained seated at rest to verify the Borg subjective perception of exertion, PA, and HR. The test was performed after a 72-hour interval of familiarization.

SPE was used to verify the individualized intensity of training ( 7 to $8=$ very easy, 9 to $10=$ easy, 11 to $12=$ relatively easy, 13 to $14=$ slightly tiring, 15 to $16=$ tiring, 17 to $18=$ very tiring, and 19 to $20=$ exhaustive), with the patients verbally encouraged to reach maximum fatigue[29].

\section{Resistance}

The resistance training protocol included 3 sets for each exercise with 12 repetitions and a one-minute interval between sets and repetitions. The speed of execution of each movement was three seconds in the concentric phase and three seconds in the eccentric phase [32]. The exercises were alternated by segment, prioritizing the large muscle groups. The loads were by means of shin guards, dumbbells, elastic bands, and the weight of the body itself.

The resistance training load was verified by means of the maximal repetition test, with 12 repetitions and a 72-hour interval of familiarization[33]. Patients who exceeded 12 repetitions were given a 5-minute interval before performing the 12 repetitions with a new load.

\section{Flexibility}

The flexibility training was active (greater range of motion performed in a contraction of the agonists and relaxation of the antagonists), without pain, where each exercise lasted 20 seconds in 3 series [34]. The flexibility exercises were: 1) Adduction of the shoulder with extension of the elbow, bilateral; 2) Shoulder and elbow flexion with palm on the back, bilateral; 3) Wrist flexion; 4) Wrist Extension; 5) Hip abduction with flexed knees; 6) Sitting, hip flexion with shoulder adduction and elbow flexion; 7) Sitting, legs stretched touching the feet; 8) Sitting, legs extended and crossed touching the feet, bilateral; 9) Shoulder flexion and adduction with hands joined in front of the trunk; 10) Back bending at the foot of the wall.

\section{Weight progression}

The load progressions were performed every 4 weeks, respecting the biological individuality in the cardiorespiratory capacity test and maximal repetitions to predict the initial load[34]. The initial intensity of the aerobic training was 50 to $60 \%$ of the THR, ending with 80 to $90 \%$ of the THR. The load of the resistance training started with the weight of the body itself or $1 \mathrm{~kg}$ in dumbbells and shin guards, and moderate intensity in the elastic band. In the fifth week there was an increase to $1 \mathrm{~kg}$ and strong intensity in the elastic band, remaining until the twelfth week (Table 1). 
Table 1

Weight progression in the combined training of patients with breast cancer.

\begin{tabular}{|c|c|c|}
\hline Weeks & Aerobic & Resistance \\
\hline $\begin{array}{l}1^{\mathrm{a}} \text { to } \\
4^{\mathrm{a}}\end{array}$ & $\begin{array}{l}50 \text { the } 60 \% \text { of } \\
\text { THR }\end{array}$ & $\begin{array}{l}\text { Body weight or } 1 \mathrm{~kg} \text { (Halters and ankle weights). elastic band (Theraband) } \\
\text { at medium setting. }\end{array}$ \\
\hline $\begin{array}{l}5^{a} \text { to } \\
8^{a}\end{array}$ & $\begin{array}{l}70 \text { the } 80 \% \text { of } \\
\text { THR }\end{array}$ & $1 \mathrm{~kg}$ and elastic band (Theraband) at high setting. \\
\hline $\begin{array}{l}9^{\mathrm{a}} \text { to } \\
12^{\mathrm{a}}\end{array}$ & $\begin{array}{l}80 \text { the } 90 \% \text { of } \\
\text { THR }\end{array}$ & Maintain intensity from 5th to 8th week. \\
\hline
\end{tabular}

\section{Blind study}

The evaluations for both the TG and CG were performed at baseline and after 12 weeks, corresponding to the intervention period. The research team was trained to apply each instrument and perform the tests, and the physical evaluations were performed blindly by the evaluator, who was only informed of the day and time of evaluations.

\section{Calculation of the sample size}

The sample size was calculated using statistical software (G-power 3.1 Düsseldorf, Germany), which showed that twelve participants were needed, through a medium effect size of 0.50 [35], a error probability of 0.05 , power (1- $\beta$ error probability) of $0.8,0.5$ correlation between repeated measure analysis (repeated measure ANOVA within-between interaction), with a nonsphericity correction of one for two groups, and two analyses throughout the study.

\section{Statistical Analysis}

Data normality was checked using the Shapiro-Wilk and Kolmogorov-Smirnov tests. The comparisons of the baseline variables between groups were analyzed using the Student $t$ test for independent samples when parametric statistics were observed. If the data presented non-parametric distribution, the MannWhitney and dichotomous variables $\chi^{2}$ and Fisher's Exact test were used.

The differences between the combined training and control groups were analyzed by two-way repeated measures of ANOVA (group x time). When a significant interaction was observed, a Bonferroni post hoc test was conducted. For all measured variables the estimated sphericity was verified according to Mauchly's W test and the Greenhouse-Geisser correction when necessary. The partial eta-squared was classified according to Cohen [35]. Statistical significance was set at $p<0.05$. The data were analyzed using the Biostatistic (Statsoft, version 10) and Stata 14.0.

\section{Results}


The original patient records contained 300 patients who could be contacted, of whom only 31 were available and participated in the sample. However, one patient in the TG was excluded due to a diagnosis of a mental disorder and another because she did not complete the physical evaluation. In the CG, one patient died and two did not complete the physical evaluation. Thus, twenty-six patients (13 TG and 13 CG) completed the study (Fig. 1).

The TG and CG patients did not present statistical differences in the anthropometric, social, demographic, and clinical variables, which presented homogeneity between the groups (Table 2). Regarding the clinical aspects, only one patient in the TG had a bilateral mastectomy and patients of neither group performed breast reconstructions. 
Table 2

Anthropometric. Social. demographic and clinics characteristics of patients with breast cancer $(n=26)$.

Variables

Antopométricas $^{a}$

Age (years)

Height (m)

Weight $(\mathrm{kg})$

Study Time

$\leq 8$ years

8 the 11 years

$>11$ years

Household income ${ }^{c}$

$\leq 2$ Minimum wages

$₫ 2$ Minimum wages

Employed ${ }^{c}$

Yes

No

Marital Status ${ }^{c}$

Single

Married

Time since most recent physical training ${ }^{c}$

3 to 12 months

$\otimes 12$ months

Clinical Period ${ }^{c}$

Observation

Chemotherapy and Hormone Therapy

Radiation Therapy

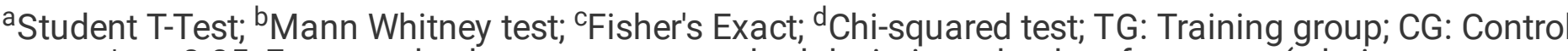
group; * $p<0.05$; Expressed values: mean \pm standard deviation. absolute frequency (relative frequency).

TG $(n=13) \quad$ CG $(n=13) \quad$ p value

$\begin{array}{lll}46.9 \pm 7.4 & 51.8 \pm 12.5 & 0.303 \\ 1.5 \pm 0.1 & 1.5 \pm 0.1 & 0.962 \\ 58.2 \pm 9.7 & 63.2 \pm 11.4 & 0.200\end{array}$

$3(23.08)$

$5(38.46)$

0.551

$7(53.85)$

$6(46.15)$

$3(23.08)$

2(15.38)

8(61.54)

10(76.92)

0.387

5(38.46)

3(23.08)

4(30.77\%)

0

0.057

9(69.23\%)

13(100\%)

7(53.85\%)

6(46.15\%)

0.848

6(46.15\%)

7(53.85\%)

4(30.77)

0

0.057

9(69.23)

13(100)

$6(46.15 \%)$

2(15.38\%)

0.343

$5(38.46 \%) \quad 8(61.54 \%)$

2(15.38\%)

3(23.08\%) 


\begin{tabular}{|c|c|c|c|}
\hline Variables & TG $(n=13)$ & CG $(n=13)$ & $p$ value \\
\hline \multicolumn{4}{|l|}{ Type of Tumor } \\
\hline Invasive Ductal Carcinoma & $13(100 \%)$ & $11(84.62 \%)$ & 0.220 \\
\hline Fuso-cellular or Epithelial Carcinoma & 0 & $2(15.38 \%)$ & \\
\hline \multicolumn{4}{|l|}{ Typo of Surgeryc } \\
\hline Conservative & $3(23.08)$ & 2(15.38) & 0.500 \\
\hline Mastectomy & $10(76.92)$ & $11(84.62)$ & \\
\hline Mastectomy + Reconstruction & 0 & 0 & \\
\hline
\end{tabular}

The body image of the patients who performed CT presented a reduction after 12 weeks for the limitations dimension ( $f=4.931, p=0.036)$, in addition to an interaction between time and group $(f=$ $10.289, p=0.003)$. In the concern with the body dimension, there was also a significant interaction $(f=$ $6.306, p=0.019)$, however, with only a tendency for TG reduction $(\Delta=5.23, f=1.876, p=0.183)$. In the transparency dimension, the groups presented significant differences $(f=8.015, p=0.009)$. The other dimensions of body image did not present significant differences (Table 3 ). 
Table 3

Body image of patients with breast cancer undergoing combined training

\begin{tabular}{|c|c|c|c|c|c|c|c|c|}
\hline \multirow[t]{2}{*}{ Dimensions } & \multicolumn{8}{|c|}{ TG $(n=13) \quad$ CG $(n=13)$} \\
\hline & Base & $\begin{array}{l}12 \\
\text { weeks }\end{array}$ & Base & $\begin{array}{l}12 \\
\text { weeks }\end{array}$ & Time & Group & Interaction & $\begin{array}{l}\text { Eta- } \\
\text { squared }\end{array}$ \\
\hline Body stigma & $\begin{array}{l}32.5 \pm \\
10.6\end{array}$ & $\begin{array}{l}33.1 \pm \\
7.7\end{array}$ & $\begin{array}{l}36.1 \pm \\
12.4\end{array}$ & $\begin{array}{l}35.6 \pm \\
14.3\end{array}$ & 0.981 & 0.478 & 0.759 & 0.00 \\
\hline Limitations & $\begin{array}{l}16.2 \pm \\
4.8\end{array}$ & $\begin{array}{l}11.2 \pm \\
2.9^{*}\end{array}$ & $\begin{array}{l}13.9 \pm \\
6.7\end{array}$ & $\begin{array}{l}14.9 \pm \\
5.7\end{array}$ & 0.036 & 0.704 & 0.003 & 0.20 \\
\hline $\begin{array}{l}\text { Concerns about } \\
\text { the arm }\end{array}$ & $\begin{array}{l}8.7 \pm \\
3.9\end{array}$ & $\begin{array}{l}9.8 \pm \\
4.1\end{array}$ & $\begin{array}{l}8.2 \pm \\
3.2\end{array}$ & $\begin{array}{l}9.8 \pm \\
3.8\end{array}$ & 0.251 & 0.773 & 0.816 & 0.05 \\
\hline Body concerns & $\begin{array}{l}17.9 \pm \\
7.0\end{array}$ & $\begin{array}{l}12.7 \pm \\
3.8\end{array}$ & $\begin{array}{l}14.3 \pm \\
6.0\end{array}$ & $\begin{array}{l}15.9 \pm \\
6.8\end{array}$ & 0.183 & 0.906 & 0.019 & 0.10 \\
\hline Transparency & $\begin{array}{l}14.7 \pm \\
5.0\end{array}$ & $\begin{array}{l}12.1 \pm \\
3.3\end{array}$ & $\begin{array}{l}17.8 \pm \\
5.2\end{array}$ & $\begin{array}{l}18.0 \pm \\
5.9\end{array}$ & 0.295 & 0.009 & 0.214 & 0.04 \\
\hline Vulnerability & $\begin{array}{l}24.2 \pm \\
9.1\end{array}$ & $\begin{array}{l}19.4 \pm \\
4.2\end{array}$ & $\begin{array}{l}21.9 \pm \\
9.6\end{array}$ & $\begin{array}{l}22.7 \pm \\
8.4\end{array}$ & 0.198 & 0.870 & 0.071 & 0.10 \\
\hline
\end{tabular}

*Difference in the TG after 12 weeks of training; TG: Training Group; CG: Control Group; Time: time referring to the intervention period; Interaction:

Referring to the interaction between time and group.

In the analysis of body composition, a change was observed after 12 weeks (time effect) for waist circumference $(f=5.019, p=0.034)$ and fat-free mass $(f=5.147, p=0.032)$, as well as a significant interaction for the circumference of the right thigh $(f=4.287, p=0.049)$. However, The strength, presented change after 12 semans, difference. Strength presented changes after 12 weeks, for both the right arm ( $f$ $=9.297, p=0.005)$, and left arm $(f=10.004, p=0.033)$ and both demonstrated a significant interaction (right $f=18.118, p=0.001$, left $f=10.004, p=0.004$ ). The post-hoc test detected increased right arm strength for the TG after 12 weeks $(p=<0.001)$. In the VO2max there was also changes after 12 weeks ( $f$ $=25.088, p=<0.001)$, in addition to a significant difference between the groups $(f=46.528, p=0.001)$. The other variables of body composition and functional capacity did not present significant differences (Table 4). 
Table 4

Body composition and functional capacity of patients with breast cancer undergoing combined training

\begin{tabular}{|c|c|c|c|c|c|c|c|c|}
\hline \multirow[t]{2}{*}{ Variables } & \multicolumn{3}{|c|}{$\begin{array}{l}\text { TG }(n= \\
13)\end{array}$} & \multirow[b]{2}{*}{$\begin{array}{l}12 \\
\text { weeks }\end{array}$} & \multirow[b]{2}{*}{ Time } & \multirow[b]{2}{*}{ Group } & \multirow[b]{2}{*}{ Interaction } & \multirow[b]{2}{*}{$\begin{array}{l}\text { Eta- } \\
\text { squared }\end{array}$} \\
\hline & Base & $\begin{array}{l}12 \\
\text { weeks }\end{array}$ & Base & & & & & \\
\hline $\begin{array}{l}\text { Body mass index } \\
\left(\mathrm{Kg} / \mathrm{m}^{2}\right)\end{array}$ & $\begin{array}{l}24.6 \\
\pm 3.7\end{array}$ & $\begin{array}{l}24.6 \\
\pm 2.9\end{array}$ & $\begin{array}{l}26.8 \\
\pm 4.0\end{array}$ & $\begin{array}{l}26.8 \\
\pm 3.9\end{array}$ & 0.864 & 0.135 & 0.947 & 0.00 \\
\hline Weight (kg) & $\begin{array}{l}58.2 \\
\pm 9.7\end{array}$ & $\begin{array}{l}58.9 \\
\pm 9.2\end{array}$ & $\begin{array}{l}63.2 \\
\pm 11.4\end{array}$ & $\begin{array}{l}63.4 \\
\pm 10.9\end{array}$ & 0.224 & 0.257 & 0.479 & 0.10 \\
\hline $\begin{array}{l}\text { Waist hip ratio } \\
(\mathrm{cm} / \mathrm{cm})\end{array}$ & $\begin{array}{l}0.8 \pm \\
0.0\end{array}$ & $\begin{array}{l}0.8 \pm \\
0.0\end{array}$ & $\begin{array}{l}0.8 \pm \\
0.1\end{array}$ & $\begin{array}{l}0.8 \pm \\
0.1\end{array}$ & 0.112 & 0.086 & 0.702 & 0.10 \\
\hline $\begin{array}{l}\text { Waist height ratio } \\
(\mathrm{cm} / \mathrm{cm})\end{array}$ & $\begin{array}{l}0.5 \pm \\
0.0\end{array}$ & $\begin{array}{l}0.5 \pm \\
0.0\end{array}$ & $\begin{array}{l}0.5 \pm \\
0.1\end{array}$ & $\begin{array}{l}0.5 \pm \\
0.1\end{array}$ & 0.259 & 0.071 & 0.689 & 0.05 \\
\hline $\begin{array}{l}\text { Conicity index } \\
(\mathrm{m} / \mathrm{kg} / \mathrm{m})\end{array}$ & $\begin{array}{l}1.2 \pm \\
0.0\end{array}$ & $\begin{array}{l}1.2 \pm \\
0.0\end{array}$ & $\begin{array}{l}1.2 \pm \\
0.2\end{array}$ & $\begin{array}{l}1.3 \pm \\
0.7\end{array}$ & 0.121 & 0.075 & 0.457 & 0.10 \\
\hline $\begin{array}{l}\text { Reciprocal ponderal } \\
\text { index }(\mathrm{cm} / \mathrm{kg})\end{array}$ & $\begin{array}{l}39.8 \\
\pm 2.0\end{array}$ & $\begin{array}{l}39.9 \\
\pm 1.6\end{array}$ & $\begin{array}{l}38.7 \\
\pm 2.0\end{array}$ & $\begin{array}{l}38.7 \\
\pm 1.8\end{array}$ & 0.845 & 0.112 & 0.860 & 0.00 \\
\hline $\begin{array}{l}\text { Percentage of fat } \\
(\%)\end{array}$ & $\begin{array}{l}28.5 \\
\pm 5.6\end{array}$ & $\begin{array}{l}27.4 \\
\pm 5.6\end{array}$ & $\begin{array}{l}28.5 \\
\pm 5.6\end{array}$ & $\begin{array}{l}27.4 \\
\pm 5.6\end{array}$ & 0.124 & 1.000 & 1.000 & 0.10 \\
\hline $\begin{array}{l}\text { Circumference of } \\
\text { the abdômen }(\mathrm{cm})\end{array}$ & $\begin{array}{l}87.1 \\
\pm 7.2\end{array}$ & $\begin{array}{l}88.2 \\
\pm 8.0\end{array}$ & $\begin{array}{l}92.2 \\
\pm 8.8\end{array}$ & $\begin{array}{l}92.7 \\
\pm 9.4\end{array}$ & 0.191 & 0.148 & 0.626 & 0.10 \\
\hline $\begin{array}{l}\text { Circumference of } \\
\text { the waist (cm) }\end{array}$ & $\begin{array}{l}77.3 \\
\pm 6.5\end{array}$ & $\begin{array}{l}78.8 \\
\pm 6.7\end{array}$ & $\begin{array}{l}82.7 \\
\pm 8.9\end{array}$ & $\begin{array}{l}83.3 \\
\pm 8.4\end{array}$ & 0.034 & 0.110 & 0.336 & 0.20 \\
\hline $\begin{array}{l}\text { Circumference of } \\
\text { the hip }(\mathrm{cm})\end{array}$ & $\begin{array}{l}96.9 \\
\pm 8.2\end{array}$ & $\begin{array}{l}97.5 \\
\pm 6.7\end{array}$ & $\begin{array}{l}100.0 \\
\pm 9.6\end{array}$ & $\begin{array}{l}99.9 \\
\pm 8.3\end{array}$ & 0.655 & 0.408 & 0.553 & 0.01 \\
\hline $\begin{array}{l}\text { Circumference of } \\
\text { the right thigh }(\mathrm{cm})\end{array}$ & $\begin{array}{l}56.2 \\
\pm 6.6\end{array}$ & $\begin{array}{l}53.7 \\
\pm 7.1\end{array}$ & $\begin{array}{l}50.9 \\
\pm 3.9\end{array}$ & $\begin{array}{l}51.9 \\
\pm 5.1\end{array}$ & 0.417 & 0.102 & 0.049 & 0.03 \\
\hline $\begin{array}{l}\text { Circumference of } \\
\text { the left thigh }(\mathrm{cm})\end{array}$ & $\begin{array}{l}54.5 \\
\pm 9.1\end{array}$ & $\begin{array}{l}52.8 \\
\pm 7.2\end{array}$ & $\begin{array}{l}50.4 \\
\pm 4.1\end{array}$ & $\begin{array}{l}51.8 \\
\pm 5.5\end{array}$ & 0.897 & 0.298 & 0.173 & 0.00 \\
\hline Fat-free mass (Kg) & $\begin{array}{l}41.3 \\
\pm 4.8\end{array}$ & $\begin{array}{l}42.4 \\
\pm 3.9\end{array}$ & $\begin{array}{l}45.0 \\
\pm 7.6\end{array}$ & $\begin{array}{l}45.8 \\
\pm 7.5\end{array}$ & 0.032 & 0.147 & 0.781 & 0.20 \\
\hline $\begin{array}{l}\text { Strength of the right } \\
\text { arm (Kgf) }\end{array}$ & $\begin{array}{l}20.2 \\
\pm 6.1\end{array}$ & $\begin{array}{l}25.7 \\
\pm 5.5^{\star}\end{array}$ & $\begin{array}{l}21.4 \\
\pm 7.4\end{array}$ & $\begin{array}{l}20.5 \\
\pm 7.1\end{array}$ & 0.005 & 0.420 & $<0.001$ & 0.30 \\
\hline $\begin{array}{l}\text { Strength of the left } \\
\text { arm (Kgf) }\end{array}$ & $\begin{array}{l}20.2 \\
\pm 6.2\end{array}$ & $\begin{array}{l}23.4 \\
\pm 5.3\end{array}$ & $\begin{array}{l}22.8 \\
\pm 6.9\end{array}$ & $\begin{array}{l}22.2 \\
\pm 6.8\end{array}$ & 0.033 & 0.765 & 0.004 & 0.20 \\
\hline
\end{tabular}

\#Difference between groups; *Difference in the TG after 12 weeks of training; TG: Training Group; CG: Control Group. Time: time referring to the intervention period; Interaction: Referring to the interaction between time and group. 


\begin{tabular}{|c|c|c|c|c|c|c|c|c|}
\hline \multirow[t]{2}{*}{ Variables } & \multicolumn{3}{|c|}{$\begin{array}{l}\text { TG }(n= \\
13)\end{array}$} & \multirow[b]{2}{*}{$\begin{array}{l}12 \\
\text { weeks }\end{array}$} & \multirow[b]{2}{*}{ Time } & \multirow[b]{2}{*}{ Group } & \multirow[b]{2}{*}{ Interaction } & \multirow[b]{2}{*}{$\begin{array}{l}\text { Eta- } \\
\text { squarec }\end{array}$} \\
\hline & Base & $\begin{array}{l}12 \\
\text { weeks }\end{array}$ & Base & & & & & \\
\hline $\begin{array}{l}\text { Maximum oxygen } \\
\text { volume }\left(\mathrm{ml}^{-1} \mathrm{~kg}^{-}\right. \\
\left.{ }^{1} \mathrm{~min}^{-1}\right)\end{array}$ & $\begin{array}{l}16.9 \\
\pm 2.0\end{array}$ & $\begin{array}{l}20.7 \\
\pm 2.6^{\#}\end{array}$ & $\begin{array}{l}11.9 \\
\pm 3.0\end{array}$ & $\begin{array}{l}14.9 \\
\pm 3.0\end{array}$ & $\hat{0} .001$ & $\hat{0} .001$ & 0.243 & 0.51 \\
\hline
\end{tabular}

At the baseline period, the CG demonstrated a strong negative correlation between waist circumference and all body image dimensions: Body Stigma (BS) $(p=0.014)$; Limitations $(L)(p=0.014)$; Concerns about the Arm (CA) ( $p=0.014)$, Body Concerns (BC) $(p=0.014)$, Transparency $(T)(p=0.014)$, and Vulnerability $(V)(p=0.014)$. The percentage of fat was positively correlated with $B C(p=0.016)$ and $T(p=0.002)$ and moderately and positively correlated with BS $(p=0.035)$ and $V(p=0.042)$. After 12 weeks, the fat percentage was still correlated strongly and positively with the BC dimension $(p=0.016)$. The muscle strength of the right arm was negatively and moderately correlated in the basal period with the $L$ dimension ( $p=0.042)$ and after 12 weeks it remained negative, but strong, for the $L$ dimension $(p=0.002)$ and moderate for the CA(0.019) and T dimensions $(p=0.035)$ (Table 5). 
Table 5

Correlation between body image, body composition and functional capacity of patients with breast cancer undergoing combined training

\section{Variables}

TG

CG

\begin{tabular}{llllllll} 
Base & & \multicolumn{2}{l}{ 12 weeks } & Base & & \multicolumn{2}{c}{12 weeks } \\
\hline $\mathrm{r}$ & $\begin{array}{l}\mathrm{p} \\
\text { value }\end{array}$ & $\mathrm{r}$ & $\begin{array}{l}\mathrm{p} \\
\text { value }\end{array}$ & $\mathrm{r}$ & $\begin{array}{l}\mathrm{p} \\
\text { value }\end{array}$ & $\mathrm{r}$ & $\begin{array}{l}\mathrm{p} \\
\text { value }\end{array}$
\end{tabular}

\section{Corporal Stigma Dimension}

Body mass index $\left(\mathrm{Kg} / \mathrm{m}^{2}\right)$

$0.03 \quad 0.925$

0.17

0.581

0.54

0.057

0.20

0.519

Weight $(\mathrm{kg})$

$\begin{array}{lll}-0.04 & 0.897 & -0.05\end{array}$

0.867

0.48

0.101

$0.16 \quad 0.612$

Waist hip ratio $(\mathrm{cm} / \mathrm{cm})$

$0.24 \quad 0.431$

0.13

0.676

$-0.05 \quad 0.865$

$0.15 \quad 0.627$

Percentage of fat (\%)

$\begin{array}{lll}-0.22 & 0.469 & -0.06\end{array}$

0.857

0.59

$0.035^{b}$

0.35

0.235

Circumference of the waist (cm)

$\begin{array}{llllllll}0.03 & 0.915 & 0.02 & 0.954 & -0.66 & \mathbf{0 . 0 1 4 ^ { \mathrm { a } }} & 0.20 & 0.509\end{array}$

Fat-free mass $(\mathrm{Kg})$

$\begin{array}{lll}0.14 & 0.649 & 0.01\end{array}$

1.000

0.21

0.489

$-0.02 \quad 0.954$

Strength of the right arm

(Kgf)

$\begin{array}{lll}-0.31 & 0.309 & -0.09\end{array}$

0.780

0.31

0.307

$-0.17 \quad 0.580$

Strength of the left arm

(Kgf)

Maximum oxygen volume

0.20

$\begin{array}{ll}-0.34 & 0.261\end{array}$

$-0.14$

0.655

0.40

0.181

$-0.06$

0.851

$\left(\mathrm{ml}^{-1} \mathrm{~kg}^{-1} \mathrm{~min}^{-1}\right)$

\section{Limitations Dimension}

\begin{tabular}{|c|c|c|c|c|c|c|c|c|}
\hline Body mass index $\left(\mathrm{Kg} / \mathrm{m}^{2}\right)$ & 0.16 & 0.610 & -0.442 & 0.131 & 0.01 & 0.998 & 0.01 & 0.991 \\
\hline Weight (kg) & 0.24 & 0.431 & -0.291 & 0.335 & 0.03 & 0.928 & 0.19 & 0.536 \\
\hline Waist hip ratio $(\mathrm{cm} / \mathrm{cm})$ & -0.02 & 0.938 & -0.459 & 0.115 & -0.15 & 0.616 & 0.04 & 0.891 \\
\hline Percentage of fat (\%) & -0.09 & 0.768 & -0.268 & 0.377 & 0.52 & 0.066 & 0.33 & 0.270 \\
\hline $\begin{array}{l}\text { Circumference of the waist } \\
(\mathrm{cm})\end{array}$ & 0.16 & 0.608 & -0.385 & 0.194 & -0.66 & $0.014^{\mathrm{a}}$ & -0.37 & 0.210 \\
\hline Fat-free mass $(\mathrm{Kg})$ & 0.33 & 0.266 & -0.239 & 0.431 & -0.22 & 0.477 & 0.03 & 0.926 \\
\hline $\begin{array}{l}\text { Strength of the right arm } \\
\text { (Kgf) }\end{array}$ & -0.41 & 0.167 & -0.233 & 0.444 & -0.57 & $0.042^{b}$ & -0.78 & $0.002^{\mathrm{a}}$ \\
\hline
\end{tabular}

aStrong and significant correlation ( $r: 0.6$ the 0.8$)$; ${ }^{b}$ Moderate and significant correlation (r:0.4 the 0.6); TG: Training Group; CG: Control Group. 


\begin{tabular}{|c|c|c|c|c|c|c|c|c|}
\hline \multirow[t]{3}{*}{ Variables } & \multicolumn{4}{|l|}{ TG } & \multicolumn{4}{|l|}{ CG } \\
\hline & \multicolumn{2}{|l|}{ Base } & \multicolumn{2}{|c|}{12 weeks } & \multicolumn{2}{|l|}{ Base } & \multicolumn{2}{|c|}{12 weeks } \\
\hline & $\mathbf{r}$ & $\begin{array}{l}\mathrm{p} \\
\text { value }\end{array}$ & $\mathbf{r}$ & $\begin{array}{l}\mathrm{p} \\
\text { value }\end{array}$ & $\mathbf{r}$ & $\begin{array}{l}p \\
\text { value }\end{array}$ & $\mathbf{r}$ & $\begin{array}{l}\mathrm{p} \\
\text { value }\end{array}$ \\
\hline $\begin{array}{l}\text { Strength of the left arm } \\
\text { (Kgf) }\end{array}$ & -0.10 & 0.739 & -0.048 & 0.877 & -0.03 & 0.900 & -0.50 & 0.080 \\
\hline $\begin{array}{l}\text { Maximum oxygen volume } \\
\left(\mathrm{ml}^{-1} \mathrm{~kg}^{-1} \mathrm{~min}^{-1}\right)\end{array}$ & 0.20 & 0.509 & 0.370 & 0.214 & -0.27 & 0.372 & 0.06 & 0.845 \\
\hline \multicolumn{9}{|l|}{$\begin{array}{l}\text { Concerns about the Arm } \\
\text { Dimension }\end{array}$} \\
\hline Body mass index $\left(\mathrm{Kg} / \mathrm{m}^{2}\right)$ & -0.27 & 0.369 & 0.11 & 0.722 & 0.50 & 0.083 & 0.09 & 0.766 \\
\hline Weight $(\mathrm{kg})$ & -0.24 & 0.426 & 0.10 & 0.733 & 0.47 & 0.106 & 0.16 & 0.604 \\
\hline Waist hip ratio $(\mathrm{cm} / \mathrm{cm})$ & 0.08 & 0.799 & -0.15 & 0.633 & 0.68 & $0.011^{a}$ & 0.11 & 0.728 \\
\hline Percentage of fat (\%) & -0.43 & 0.145 & 0.14 & 0.648 & -0.33 & 0.264 & 0.14 & 0.653 \\
\hline $\begin{array}{l}\text { Circumference of the waist } \\
(\mathrm{cm})\end{array}$ & -0.27 & 0.378 & 0.12 & 0.699 & -0.66 & $0.014^{a}$ & -0.37 & 0.210 \\
\hline Fat-free mass (Kg) & -0.05 & 0.868 & 0.07 & 0.831 & 0.67 & $0.011^{\mathrm{a}}$ & 0.09 & 0.759 \\
\hline $\begin{array}{l}\text { Strength of the right arm } \\
\text { (Kgf) }\end{array}$ & -0.33 & 0.265 & 0.20 & 0.510 & 0.06 & 0.844 & -0.64 & $0.019^{a}$ \\
\hline $\begin{array}{l}\text { Strength of the left arm } \\
\text { (Kgf) }\end{array}$ & -0.24 & 0.432 & 0.08 & 0.801 & 0.09 & 0.781 & -0.41 & 0.159 \\
\hline $\begin{array}{l}\text { Maximum oxygen volume } \\
\left(\mathrm{ml}^{-1} \mathrm{~kg}^{-1} \mathrm{~min}^{-1}\right)\end{array}$ & 0.20 & 0.509 & 0.28 & 0.361 & -0.64 & $0.018^{a}$ & -0.16 & 0.600 \\
\hline \multicolumn{9}{|l|}{ Body Concerns Dimension } \\
\hline Body mass index $\left(\mathrm{Kg} / \mathrm{m}^{2}\right)$ & -0.10 & 0.739 & 0.01 & 0.964 & 0.27 & 0.373 & 0.01 & 0.964 \\
\hline Weight (kg) & -0.10 & 0.741 & 0.14 & 0.638 & 0.37 & 0.218 & 0.13 & 0.678 \\
\hline Waist hip ratio $(\mathrm{cm} / \mathrm{cm})$ & 0.38 & 0.198 & 0.18 & 0.554 & -0.04 & 0.897 & -0.09 & 0.765 \\
\hline Percentage of fat (\%) & -0.37 & 0.216 & 0.05 & 0.868 & 0.65 & $0.016^{\mathrm{a}}$ & 0.65 & $0.016^{a}$ \\
\hline $\begin{array}{l}\text { Circumference of the waist } \\
(\mathrm{cm})\end{array}$ & -0.03 & 0.927 & 0.10 & 0.751 & -0.66 & $0.014^{a}$ & -0.37 & 0.210 \\
\hline Fat-free mass (Kg) & 0.14 & 0.649 & 0.17 & 0.586 & 0.08 & 0.790 & -0.18 & 0.563 \\
\hline
\end{tabular}


CG

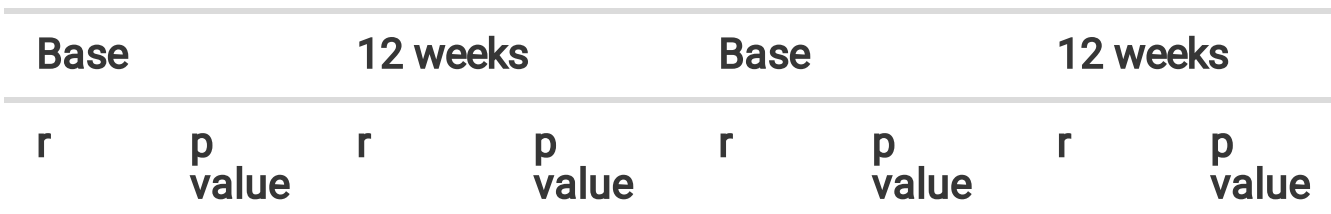

Strength of the right arm (Kgf)

$-0.20$

$0.509 \quad-0.47$

0.106

$-0.29$

0.337

$-0.25 \quad 0.420$

Strength of the left arm (Kgf)

$\begin{array}{llllllll}-0.20 & 0.522 & -0.21 & 0.482 & -0.03 & 0.931 & -0.22 & 0.473\end{array}$

Maximum oxygen volume

0.20

0.509

0.28

0.361

$-0.37$

0.220

0.25

0.246

$\left(\mathrm{ml}^{-1} \mathrm{~kg}^{-1} \mathrm{~min}^{-1}\right)$

\section{Transparency Dimension}

\begin{tabular}{lcccccccc} 
Body mass index $\left(\mathrm{Kg} / \mathrm{m}^{2}\right)$ & 0.10 & 0.757 & 0.37 & 0.211 & 0.27 & 0.364 & -0.06 & 0.834 \\
\hline Weight $(\mathrm{kg})$ & -0.08 & 0.789 & 0.28 & 0.360 & 0.27 & 0.371 & 0.04 & 0.889 \\
\hline Waist hip ratio $(\mathrm{cm} / \mathrm{cm})$ & 0.31 & 0.302 & -0.24 & 0.434 & -0.10 & 0.745 & 0.05 & 0.866 \\
\hline $\begin{array}{l}\text { Percentage of fat (\%) } \\
\text { Circumference of the waist }\end{array}$ & 0.07 & 0.828 & 0.45 & 0.126 & 0.78 & $0.002^{\mathrm{a}}$ & 0.14 & 0.647 \\
\hline $\begin{array}{l}\text { (cm) } \\
\text { Fat-free mass }(\mathrm{Kg})\end{array}$ & -0.15 & 0.624 & 0.18 & 0.563 & -0.66 & $0.014^{\mathrm{a}}$ & -0.37 & 0.210 \\
\hline $\begin{array}{l}\text { Strength of the right arm } \\
\text { (Kgf) }\end{array}$ & 0.02 & 0.953 & 0.45 & 0.125 & -0.17 & 0.574 & -0.59 & $0.035^{\mathrm{b}}$ \\
\hline $\begin{array}{l}\text { Strength of the left arm } \\
\text { (Kgf) }\end{array}$ & 0.01 & 0.996 & 0.26 & 0.392 & 0.08 & 0.795 & -0.35 & 0.242 \\
\hline
\end{tabular}

$\begin{array}{lllllllll}\text { Maximum oxygen volume } & 0.20 & 0.509 & 0.28 & 0.361 & -0.30 & 0.328 & 0.05 & 0.869\end{array}$ $\left(\mathrm{ml}^{-1} \mathrm{~kg}^{-1} \mathrm{~min}^{-1}\right)$

\section{Vulnerability Dimension}

a Strong and significant correlation ( $r: 0.6$ the 0.8$)$; ${ }^{b}$ Moderate and significant correlation ( $r: 0.4$ the 0.6$)$; TG: Training Group; CG: Control Group. 


\begin{tabular}{|c|c|c|c|c|c|c|c|c|}
\hline \multirow[t]{3}{*}{ Variables } & \multicolumn{4}{|l|}{ TG } & \multicolumn{4}{|l|}{ CG } \\
\hline & \multicolumn{2}{|l|}{ Base } & \multicolumn{2}{|c|}{12 weeks } & \multicolumn{2}{|l|}{ Base } & \multicolumn{2}{|c|}{12 weeks } \\
\hline & $\mathbf{r}$ & $\begin{array}{l}\mathrm{p} \\
\text { value }\end{array}$ & $\mathbf{r}$ & $\begin{array}{l}\mathrm{p} \\
\text { value }\end{array}$ & $\mathbf{r}$ & $\begin{array}{l}\mathrm{p} \\
\text { value }\end{array}$ & $\mathbf{r}$ & $\begin{array}{l}\mathrm{p} \\
\text { value }\end{array}$ \\
\hline Body mass index $\left(\mathrm{Kg} / \mathrm{m}^{2}\right)$ & -0.29 & 0.341 & -0.61 & $0.027^{a}$ & 0.22 & 0.463 & 0.20 & 0.519 \\
\hline Weight (kg) & -0.37 & 0.216 & -0.63 & $0.021^{a}$ & 0.28 & 0.362 & 0.31 & 0.302 \\
\hline Waist hip ratio $(\mathrm{cm} / \mathrm{cm})$ & 0.18 & 0.564 & -0.64 & $0.018^{a}$ & 0.06 & 0.851 & -0.01 & 0.963 \\
\hline Percentage of fat (\%) & -0.33 & 0.275 & -0.41 & 0.169 & 0.57 & $0.042^{b}$ & 0.38 & 0.197 \\
\hline $\begin{array}{l}\text { Circumference of the waist } \\
\text { (cm) }\end{array}$ & -0.24 & 0.428 & -0.61 & $0.026^{a}$ & -0.66 & $0.014^{a}$ & -0.37 & 0.210 \\
\hline Fat-free mass (Kg) & -0.26 & 0.383 & -0.68 & $0.011^{a}$ & 0.01 & 0.981 & 0.12 & 0.696 \\
\hline $\begin{array}{l}\text { Strength of the right arm } \\
\text { (Kgf) }\end{array}$ & -0.43 & 0.139 & -0.20 & 0.510 & -0.38 & 0.199 & -0.54 & 0.058 \\
\hline $\begin{array}{l}\text { Strength of the left arm } \\
\text { (Kgf) }\end{array}$ & -0.43 & 0.141 & -0.12 & 0.689 & 0.16 & 0.598 & -0.24 & 0.434 \\
\hline $\begin{array}{l}\text { Maximum oxygen volume } \\
\left(\mathrm{ml}^{-1} \mathrm{~kg}^{-1} \mathrm{~min}^{-1}\right)\end{array}$ & -0.46 & 0.119 & 0.28 & 0.361 & -0.55 & 0.053 & 0.21 & 0.496 \\
\hline
\end{tabular}

The concern with the arm dimension in the CG presented different correlations with the other dimensions, such as: a positive and strong correlation with the waist-hip ratio $(p=0.011)$ and fat-free mass $(p=$ $0.011)$, and negative correlation with the volume of oxygen $(p=0.018)$ in the basal period (Table 5$)$.

The TG did not present any correlations between variables of functional capacity and body image. On the other hand, body composition variables, specifically the body mass index $(p=0.027)$, weight $(0.021)$, waist-hip ratio $(p=0.018)$, waist circumference $(p=0.026)$, and fat-free mass $(p=0.011)$ were negatively correlated with vulnerability, in the pre-training, but gained statistical significance only after the intervention.

\section{Discussion}

The main findings of this research can be summarized as follows: 1) CT promoted improvements in functional capacity, but not in body composition in TG women; 2) CT favored positive changes in body image; 3) Variables related to appearance were correlated with the vulnerability dimension in TG;4) Variables related to body appearance and function were directly related to body image in the CG. The 
latter was our most surprising finding, particularly the waist circumference, which appears to be an important marker in the body image of women with breast cancer not submitted to physical activity.

The effect of CT was confirmed by increased arm muscle strength and improvements in VO2max. These results corroborate studies in the literature which submitted women with breast cancer to protocols composed of aerobic and strength exercises and found similar results [20-22]. CT prevents the physical deconditioning inherent in cancer treatment [22]. These variables enhance the perception of this population of improvements in their quality of life [18]. De Luca et al.[21] further point out that CT promotes greater adherence to physical activity, due to the diversification of exercises.

However, in the current study, there were no positive changes in body mass, percentage of fat, BMI, or other anthropometric indices in either group. It is worth noting that, despite this, the maintenance of these variables already indicates a good follow-up, since the disease and its treatment promote negative changes in body composition[7]. Similar results were found in women with breast cancer submitted to strength training [18], aerobic[17] and combined (strength and aerobic) protocols [20]. On the other hand, De Luca et al. [21] observed a reduction in fat percentage after intervention with CT in the same population. This difference may be attributed to the superiority in the intervention time (24 weeks) and method of analysis (bioimpedance), performed by the researchers. However, the multi-frequency electrical bioimpedance analysis method indicates greater precision when performed in a segmental way, due to the morphological variation in the tissues[36].

On the other hand, there was an interaction between time and group for the right thigh and changes in fatfree mass and waist circumference after 12 weeks. Despite the maintenance of these variables, promoting health through physical training, there was an increase in the waist circumference (WC) of both groups, which shows a negative trend and can be attributed to the cancer treatment. According to Oliveira et al. [37] and Figueiredo et al. [38] there is a large incidence of high WC in patients with breast cancer, which is linked to cardiovascular risk; these authors suggest that this population requires adherence to a nutritional program. However, the study by Kim et al. [39] demonstrated a reduction in WC after a 12-week CT intervention, this difference may be due to the stage of the treatment, since all the patients were survivors of breast cancer. Further studies are needed to define the type of intervention effective in the reduction of visceral fat in patients in the treatment of breast cancer.

Another central axis of this research is the investigation of body image changes. This was analyzed from the theoretical model with six dimensions[23] and validated for Brazilian women with breast cancer[5]. Three dimensions demonstrated sensitivity to CT: limitation, concern for the body, and transparency. After the intervention, the TG demonstrated a significant reduction in the perception of functional limitations of the body, such as movement restrictions and oncologic fatigue. Arab et al. [16] in one of the few studies in this scope performed in Brazil, presented similar results, although the protocol applied by them was different, with only resistance training over 12 weeks. The authors attribute the improvement to the higher physical competence acquired for the performance of motor tasks. Unlike our data, Arab et al. [16] did not 
find training effects in the other dimensions [23]. It is possible that this difference is associated with the specificity of the intervention given.

Concern with the body is a striking feature in women with breast cancer, which in Brazilian women (who culturally already have this concern)[40], is accentuated either through the chemotherapy or mastectomy process [41]. As we hypothesized, the TG showed a tendency to reduce concerns with the body, that is, with their general appearance, including concern about the gain or loss of weight. Previous studies have demonstrated similar results after both strength training[19] and aerobic training[17], in which improvements in the perception of body appearance and lower concern with weight were detected, respectively.

Concern with appearance is related to the alterations promoted by the disease and treatment, which may be less or more visible[23]. Issues relating to concern with how obvious the disease is (expressed by changes in appearance) were denominated transparency by the authors. This variable was significantly different between the groups, so that women who did not receive the intervention with the CT presented higher scores in this dimension. We did not find similar studies that addressed this point, which limits our discussion. However, a qualitative research with Latina women with breast cancer, identified that the acceptance of changes in appearance is considered a central axis in body image [11]. The authors encourage the development of intervention strategies that favor the acceptance of appearance during and after treatment. Our results suggest that CT may be of potential assistance. This becomes more consistent when we observe the gross scores of all analyzed dimensions, and find that, although there is no statistical significance, there is an increase in the $C G$ and reduction in the $T G$, indicating a tendency to reduce body image impairment with the practice of CT[5].

In the same direction, the next question was to identify whether improvements in body image could be attributed to changes in appearance and/or functionality as a result of CT. This hypothesis was partially rejected because the TG did not present a significant correlation between body image and functional capacity. On the other hand, the body composition, BMI, weight, WHR, WC, and fat-free mass variables, although not presenting significant changes in our sample, were negatively correlated with body image, specifically with the vulnerability dimension, in the basal period, assuming statistical significance after the intervention. This fact leads us to reflect that the body experience with the CT may have directed the attention of these women to their body measurements, however, differently to women without breast cancer, since the literature indicates that BMI, WHR, WC, and fat-free mass are predictors of negative changes in body image, such as body dissatisfaction[42, 40]. In our study, the opposite occurred, the higher these scores, the lower the feeling of invasion of the body by the disease, which may have consequently caused a lower sensation of vulnerability.

Unlike our results, Speck et al.[19]and Pinto et al.[17], after interventions with strength and aerobic training, respectively, in women with breast cancer, did not identify any variable of body composition and/or function capable of mediating the effect of training on the improvements found in body image. On the other hand, the positive effect on functional capacity, identified here and in the studies above, is 
pointed out by the authors as a factors that influences body image, although indirectly. Speck et al. [19] explain that muscular strength provides benefits to the general quality of life and this, in turn, mediates the intervention in the perception of the body. Pinto et al. [17] concluded that the improvement found in patients' self-assessment of their physical condition (energy, strength, and agility) is consistent with the increase in VO2max, thus indicating a refinement of the patient about her physical condition.

In contrast to the TG, the body image of the women who were not submitted to the intervention was influenced, over time, as much by the variables related to appearance as by body functionality. Waist circumference, a variable commonly associated positively with female body dissatisfaction[40], precisely because it delineates female body forms, manifested itself in an opposite way for all dimensions of body image. Thus, the smaller this variable, the greater the perception of functional limitations, the concern with the arm and with the body, accentuating the feelings of vulnerability, visibility of the disease (transparency), and body stigma.

The percentage of fat, also considered a predictor of body dissatisfaction in women[40], especially for the lean body ideal[42], maintained this characteristic for the CG, in proportion to the Body Stigma, body concerns, transparency, and vulnerability. It is possible that this is due to the gradual and complex process of acceptance of changes in appearance from disease and treatment, requiring women to learn and deal with these changes[11].

Functional ability also presented an influence on CG body image. The CG showed a negative correlation between arm muscle strength and the limitations and transparency dimensions (despite the absence of significance in the basal period) in all phases. Concerns with the arm were positively related (although not significant at baseline) to muscle strength, assuming statistical significance, but negative, after 12 weeks. The opposite occurred between concerns about the arm and VO2max. Ohira et al.[43], when developing research similar to ours, hypothesized that women with breast cancer feel empowered psychologically as they become more physically effective. Although we cannot state that the benefits of CT positively and directly impacted body image in the TG, the authors' idea applies in our results, since we observed that impairments in functional capacity were negatively associated with CG body image.

However, we believe that, unlike the CG, the TG may have benefited from body experiences in the intervention, thus impacting dimensions which, although not evaluated herein, are indicated in the specific literature as linked to this process: cognitive, affective, and behavioral[10]. Interventions with physical exercise can provide the sensation of regaining control of the body itself, which may translate into a greater sense of self-efficacy in other areas of life [43]. Thus, it is possible to infer that CT promotes subjective experiences that go beyond body appearance and function, although indirectly influencing it.

Finally, we agree with the point of view of Speck et al.[19], in which the authors point out that the mechanism of impact of training on the concern with the body of women with breast cancer remains unclear. Thus, we encourage researchers to engage in research of this scope that seeks to identify whether CT can be considered a protective element for negative changes in body image in this population. In addition to this gap, we also recognize some methodological limitations of this research. 
Initially, the sample size is restricted, which hampers generalizations of the results. The high exclusion of participants may negatively impact the results of randomized clinical trials, biasing the research. In addition, the history of physical exercise of non-eligible patients was not investigated, information that may be useful for understanding some results. Finally, the reduced number of articles with the same theme in Brazilian women affected by breast cancer made the discussion difficult, since cultural factors are extremely relevant to the body image constructs [10].

Although we analyzed important variables in the elaboration of the body image of this population, such as the type of surgery $[13,4,44]$, a relevant point in this context is breast reconstruction, since it is known that women undergoing reconstruction are less dissatisfied with their bodies[41]. Accordingly, we recommend new studies that compare women with and without breast reconstruction and analyze the relationship of body image with body composition and functional capacity.

\section{Conclusion}

Combined training was shown to be a useful strategy capable of promoting improvements in the functional capacity and body image of women with breast cancer. We recommend its use together with conventional treatment. Thus, the data obtained here have theoretical and practical implications. The first refers to the need to broaden the understanding of body image adaptations as a function of specific physical changes in breast cancer, using a specific tool and theoretical axis that considers body image as a multidimensional and independent construct.

We believe that this information could help in the delineation of facilitating factors, mediators, and protectors of body image in the treatment process, allowing the elaboration of adequate interventions. Practical implications involve the use of this information in interventions that deal directly with the body, making them more assertive and efficient.

\section{Abbreviations}

Combined Training - CT; Training Group - TG; Control Group - CG; Vulnerability - V; Transparency - T; Body Stigma (BS); Concerns about the Arm (CA), Body concerns - BC; Limitations - L; Body Mass Index - BMl; Waist-hip ratio - WHR; Waist Height Ratio- RCEST; Conicity Index - C Index; Reciprocal Ponderal Index - RPI; Percentage of Fat - \%F; Body Density - BD; maximum oxygen volume - VO2max; American College of Sports Medicine - ACSM; training heart rate - THR; subjective perception of exertion - SPE; Foundation for the Support of Scientific Research and Development of Maranhão- FAPEMA; Coordination of Improvement of Higher Level Personnel- CAPES; Laboratory of Physical Assesssment and Psychomoter and Whole Body Rehabilitation- LAREPO; Laboratory of Physiology and Prescribed Exercise of Maranhão- LAFIPEMA; Pain Management League - LAD and Aldenora Bello Cancer Hospital in São Luís-MA.

\section{Declarations}




\section{Ethics approval and consent to participate:}

The study is accordance with the Declaration of Helsinki and had approval from the Research Ethics Committee of the Federal University of Maranhão, protocol number 20665713.2.0000.5087. The Term of Free and Informed Consent was informed in writing by all participants.

\section{Consent for publication:}

The Term of Free and Informed Consent was applied to all patients in the study. But including any individual details, images or videos are not applicable.

\section{Competing interests:}

The authors declare that there are no conflicts of interest. The results of the study is presented clearly, honestly, and without fabrication, falsification, or inappropriate data manipulation. The authors declare that they have no competing interests.

\section{Availability of Data and Materials:}

The datasets used and/or analysed during the current study are available from the corresponding author on reasonable request.

\section{Funding:}

Not applicable.

\section{Authors' contributions:}

ADR wrote the project, conducted the research, wrote the manuscript, analyzed and interpreted the data; PTVTP conducted the research and wrote the manuscript; JGLCF wrote the project and conducted the research; EFR conducted the research and wrote the manuscript; IPL analyzed and interpreted the data; BTR conducted the research and wrote the manuscript; MRdC wrote the manuscript and reviewed the manuscript; FER analyzed and interpreted the data; IFFJ analyzed and interpreted the data; JBSG: wrote the project, reviewed the manuscript, guided, analyzed and interpreted the data.

\section{Acknowledgements:}


Thank you to the Foundation for the Support of Scientific Research and Development of Maranhão FAPEMA, Coordination of Improvement of Higher Level Personnel - CAPES, Laboratory of Physical Assesssment and Psychomoter and Whole Body Rehabilitation - LAREPO, Laboratory of Physiology and Prescribed Exercise of Maranhão - LAFIPEMA, Pain Management League - LAD and Aldenora Bello Cancer Hospital in São Luís-MA.

\section{References}

1. World Health Organization. Cancer fact sheet WHO. (2016). Accessed 18th Dezember, 2018.

2. Ferlay J, Soerjomataram I, Dikshit R, Eser S, Mathers C, Rebelo M, et al. Cancer incidence and mortality worldwide: Sources, methods and major patterns in GLOBOCAN 2012. Int J Cancer 2015, 136(5); doi:10.1002/ijc.29210.

3. Instituto Nacional de Câncer José Alencar Gomes da Silva. Estimativa 2018: Incidência de câncer no Brasil. INCA. Rio de Janeiro, INCA, 2017.

4. Rosenberg SM, Tamimi RM, Gelber S, Ruddy KJ, Kereakoglow S, Borges VF, et al. Body image in recently diagnosed young women with early breast cancer. Psycho-Oncology. 2013, 22(8); doi:10.1002/pon.3221.

5. Gonçalves CO, Tavares MCGCF, Campana ANNB, Cabello C. Validation of the instrument "Body image after breast cancer" in Brazil. Motriz 2014, 20(1); doi:dx.doi.org/10.1590/S198065742014000100002.

6. Marques JR, Martins PCML, Machado ER, Souza LMde, Rodrigues JHA. Análise dos efeitos da drenagem linfática manual no tratamento do linfedema pós mastectomia. Revista Acadêmica do Instituto de Ciências da Saúde. 2015, 1(1):72-82.

7. Rubin BA, Stein AT, Zelmanowicz AM, Rosa DD. Perfil Antropométrico e Conhecimento Nutricional de Mulheres Sobreviventes de Câncer de Mama do Sul do Brasil. Revista Brasileira de Cancerologia. 2010;56(3):303-9.

8. Ewertz M, Jensen MB, Gunnardóttir K, Hojris I, Jakobsen EH, Nielsen D, et al. Effect of obesity on prognosis after early-stage breast cancer. J of Clinical Oncology. 2011, 29(1); doi:10.1200/JC0.2010.29.7614.

9. Muzzatti B, Annunziata MA. Body image assessment in oncology: An update review. Support Care Cancer. 2017, 25(3); doi:10.1007/s00520-016-3538-y.

10. Cash TF, Smolak L. Body image: A handbook of science, practice and prevention. Ed. New York: Guilford Press; 2011.

11. Buki LP, Reich M, Lehardy EN. "Our organs have a purpose": Body image acceptance in latina breast cancer survivors. Psychooncology 2016, 25(11); doi:10.1002/pon.4270.

12. Fingeret MC, Teo I, Epner DE. Managing body image difficulties of adult cancer patients: lessons from available research. Cancer. 2014, 120(5); doi: 10.1002/cncr.28469. 
13. Paterson CL, Lengacher CA, Donovan KA, Kip KE, Tofthagen CS. Body image in younger breast cancer survivors: A systematic review. Cancer Nursing 2016, 39(1); doi:10.1097/NCC.0000000000000251.

14. Schmitz KH, Courneya KS, Matthews C, Demark-Wahnefried W, Galvão DA, Pinto BM, et al. American College of Sports Medicine roundtable on exercise guidelines for cancer survivors. Med Sci Sports Exerc. 2010, 42(7); doi:10.1249/MSS.0b013e3181e0c112.

15. Hornsby WE, Douglas PS, West MJ, Kenjale AA, Lane AR, Schwitzer ER, et al. Safety and efficacy of aerobic training in operable breast cancer patients receiving neoadjuvant chemotherapy: a phase II randomized trial. Acta Oncol. 2014, 53(1); doi:10.3109/0284186X.2013.781673.

16. Arab C, Angarten VG, Crocetta TB, Sorpreso ICE, Abreu LCde, Andrade A. Resistance exercise program during breast cancer treatment: A feasibility study. International Archives of Medicine 2016, 9(201); doi:dx.doi.org/10.3823/2072.

17. Pinto BM, Clark MM, Maruyama NC, Feder SI. Psychological and fitness changes associated with exercise participation among women with breast cancer. Psycho-Oncology. 2003, 12(2); doi:10.1002/pon.618.

18. Benton MJ, Schlairet MC, Gibson DR. Change in quality of life among breast cancer survivors after resistance training: Is there an effect of age? J of Aging and Physical Activity. 2014, 22(2); doi: dx.doi.org/10.1123/JAPA.2012-0227.

19. Speck RM, Gross CR, Hormes JM, Ahmed RL, Lytle LA, Hwang WT, et al. Changes in the body image and relationship scale following a one-year strength training trial for breast cancer survivors with or at risk for lymphedema. Breast Cancer Res Treat. 2010, 121(2); doi:10.1007/s10549-009-0550-7.

20. Ortega JAF, Fernandez JAP. Effects of a combined strength and high-intensity aerobic exercise program in breast câncer survivors: a pilot study. Apunts Medicina de l'Esport 2016, 51(189); doi:doi.org/10.1016/j.apunts.2015.10.003.

21. De Luca V, Minganti C, Borrione P, Grazioli E, Cerulli C, Guerra E, et al. Effects of concurrent aerobic and strength training on breast cancer survivors: a pilot study. Public Health. 2016, 136; doi:10.1016/j.puhe.2016.03.028.

22. Travier N, Velthuis MJ, Steins Bisschop CN, Van den Buijs B, Monninkhof EM, Backx F, et al. Effects of an 18-week exercise programme started early during breast cancer treatment: A randomised controlled trial. BMC Med. 2015, 13(1); doi:10.1186/s12916-015-0362-z.

23. Baxter NN, Goodwin PJ, McLeod RS, Dion R, Devins G, Bombardier C. Reliability and validity of the body image after breast cancer questionnaire. Breast J. 2006 12(3); doi: 10.1111/j.1075122X.2006.00246.X.

24. Gonçalves CO, Tavares MCGCF, Campana ANNB, Cabello C, Shimo AKK. Instruments to evaluate body image in women with breast cancer. Psicol Teor Prat. 2012;14(2):43-55.

25. Peres ACAM. Avaliação da postura, qualidade de vida, imagem corporal e autoestima em mulheres com mastectomia sem reconstrução e com a reconstrução imediata da mama. São Paulo. 
Dissertação. [Mestre em Ciências] - Universidade de São Paulo; 2014. Doi: 10.11606/D.5.2014.tde05082014-154238.

26. American College of Sports Medicine. ACSM's guidelines for exercise testing and prescription. Guanabara Koogan: Ed. Rio de Janeiro; 2003.

27. Caminha TCS, Harold SF, Narithania SC, Nakano RP, Carvalho RES, Xavier AFS Jr, et al. Waist-toheight ratio is the best anthropometric predictor of hypertension. Medicine. 2017, 96(2); doi:10.1097/MD.0000000000005874.

28. Damasceno VO, Dutra LN, Ribeiro LG, Vianna VRA, Vianna JM, Novaes JS, et al. Body mass index and reciprocal of ponderal index as predictors of overwight and obesity. Rev. Bras de Cineant $\mathrm{e}$ Desemp Humano. 2003;5(2):44-52.

29. Fontoura AS, Formentin CM, Abech EA. Guia prático de avaliação física. Uma abordagem didática, abrangente e atualizada. Ed. 2 revisada e ampliada. Phorte: São Paulo; 2013.

30. Karvonen MJ, Kentala E, Mustala $\mathrm{O}$. The effects of training on heart rate; a longitudinal study. Annales Medicina e Experimentalis et Biologiae Fenniae Helsinki. 1957;35(3):307-15.

31. Niel SE, Klika RJ, Garland SJ, McKenzie DC, Campbell KL. Cardiorespiratory and neuromuscular deconditioning in fatigued and non-fatigued breast cancer survivors. Support Care Cancer. 2013 21(3); doi:10.1007/s00520-012-1600-y.

32. Battaglini C, Bottaro M, Dennehy C, Barfoot D, Shields E, Kirk D, et al. The effects of resistance training on muscular strength and fatigue levels in breast cancer patients. Rev Bras de Med do Esporte. 2006, 12(3); doi:dx.doi.org/10.1590/S1517-86922006000300009.

33. Guedes DPE, Guedes JERP. Manual prático para avaliação em educação física. ed. 1. Manole: São Paulo; 2006.

34. Weineck J. Treinamento ideal. ed.9. Manole: São Paulo; 2003.

35. Cohen J. Statistical Power Analysis for the Behavioral Sciences. 2nd ed. New York: Lawrence Erlbaum Associates; 1988.

36. Cornish BH, Eles PT, Thomas BJ, Ward LC. The effect of electrode placement in measuring ipsilateral/contralateral segmental bioelectrical impedance. Lymphatic Research Biology. 2006, 4(1); doi:10.1111/j.1749-6632.2000.tb06455.x.

37. Oliveira DR, Carvalho ESC, Campos LC, Leal JA, Sampaio EV, Cassali GD. Avaliação nutricional de pacientes com câncer de mama atendidas no Serviço de Mastologia do Hospital das Clínicas, Belo Horizonte (MG), Brasil. Ciência \& Saúde Coletiva. 2014, 19(5); doi: 10.1590/141381232014195.02262013.

38. Figueiredo ACDS, Saço LF, Damasceno VO, Ferreira RNF, Ferreira EL. Associação entre variáveis antropométricas e o tratamento para o câncer de mama. ConScientiae Saúde. 2014, 13(1); doi:10.5585/ConsSaude.v13n1.4783.

39. Kim TH, Chang JS, Park KS, Park J, Kim N, Lee Jl, et al. Effects of exercise training on circulating levels of Dickkpof-1 and secreted frizzled-related protein-1 in breast cancer survivors: A pilot singleblind randomized controlled trial. PloS one. 2017, 12(2); doi:10.1371/journal.pone.0171771. 
40. Laus MF, Miranda VP, Almeida SS, Braga Costa TM, Ferreira ME. Geographic location, sex and nutritional status play an important role in body image concerns among Brazilian adolescents. J of Health Psychology. 2017, 12(2); doi:10.1177/1359105311434755.

41. Prates ACL, Freitas-Junior R, Prates MFO, Veloso MF, Barros NM. Influence of Body Image in Women Undergoing Treatment for Breast Cancer. Rev Bras Ginecol Obstet. 2017, 39(4); doi:10.1055/s-00371601453.

42. Stewart A, Crockett P, Nevill A, Benson P. Somatotype: a more sophisticated approach to body image work with eating disorder sufferers. Advances in Eating Disorders: Theory Research Practice. 2014, 2(2); doi:10.1080/21662630.2013.874665.

43. Ohira T, Schmitz KH, Ahmed RL, Yee D. Effects of weight training on quality of life in recent breast cancer survivors: The weight training for breast cancer survivors (WTBS) study. Cancer. 2006, 106(9); doi:10.1002/cncr.21829.

44. Chow KM, Hung KL, Yeung SM. Body image and quality of life among breast cancer survivors: $A$ literature review. World Journal of Oncology Research. 2016, 3; doi:dx.doi.org/10.15379/24137308.2016.03.02.

\section{Figures}

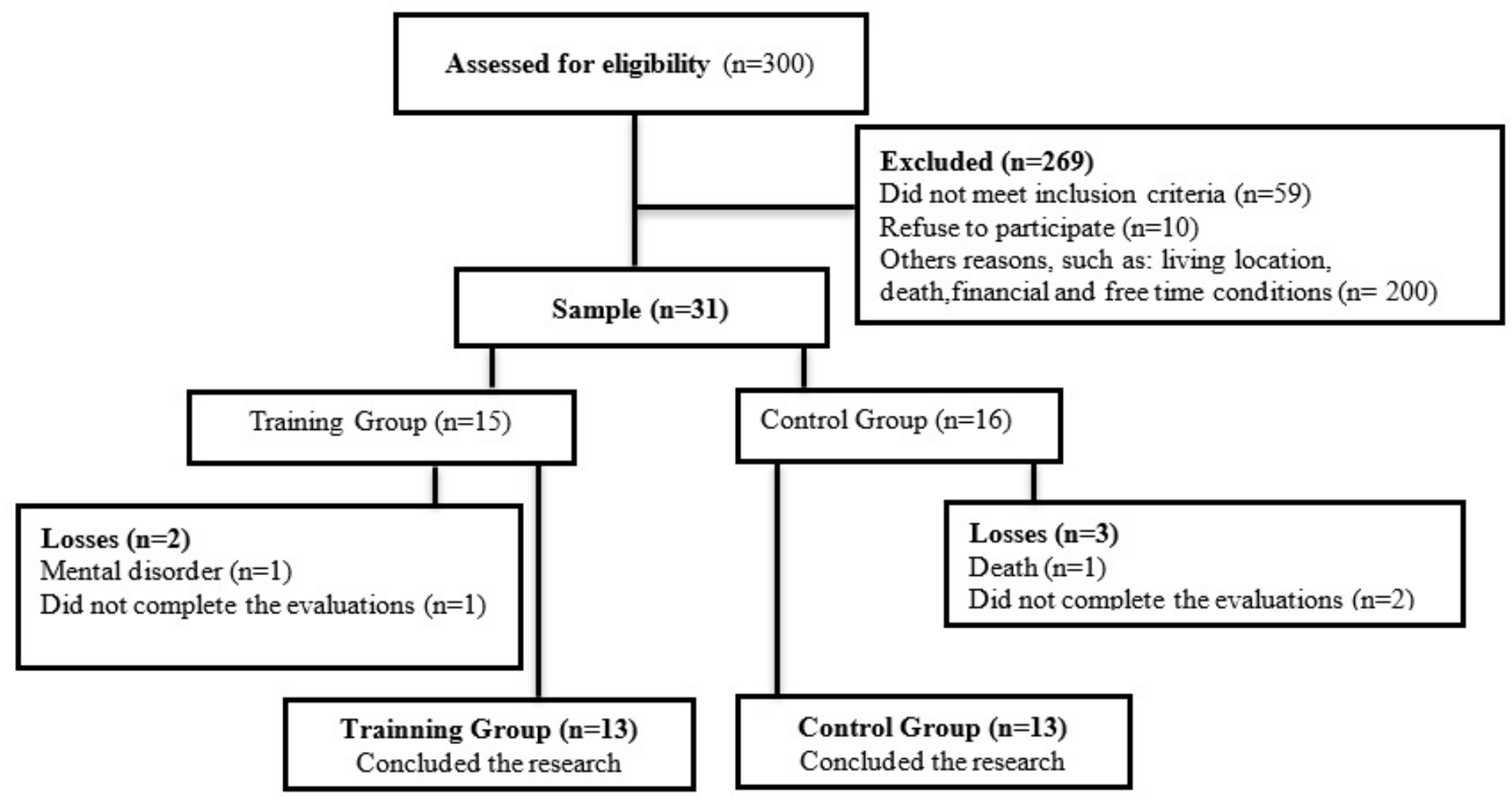

Figure 1

Consort flowchart. 


\section{Supplementary Files}

This is a list of supplementary files associated with this preprint. Click to download.

- TrialRegistration.pdf

- CONSORT2010Checklist.pdf 